Introduction: Climate change and overdevelopment increase the intensity and frequency of flash flooding, which may generate more swiftwater rescue (SWR) incidents. Rescue personnel may fail to properly risk stratify (triage) these victims due to limited medical and/or variable SWR training, or due to an adverse rescuer-to-victim ratio. Some victims may attempt to refuse medical evaluation due to lack of awareness of incident-related morbidity and/or comprehension of risk.

Aim: To develop an SWR emergency medical triage tool. Methods: A cross-sectional literature search identified SWRrelated medical conditions. A flow diagram reliant upon incident history, chief complaint, and observational exam rather than interpretation of vital signs was created to guide medical decision-making.

Results: Every SWR victim should receive a medical screening exam focused on six clinical categories-drowning, hypothermia, hazmat exposure, physical trauma, psychological trauma and exacerbation of pre-existing disease. Drowning potential is identified by dyspnea, new cough or a history of (even brief) submersion. Shivering SWR victims and those with altered mental status but no shivering are assumed to be hypothermic. Any victim with open skin lesions/wounds who was immersed in floodwater and anyone who has swallowed floodwater is contaminated; these victims require decontamination and possible antibiotic therapy. SWR victims injured upon entering the water or from contact with either water-borne stationary or floating objects require trauma evaluation. Distraught victims and those who exhibit exacerbation of pre-existing organsystem disease also require ED evaluation.

Discussion: Most SWR course curricula are oriented towards technical rescue; they do not address comprehensive medical decision-making. We present a rapid medical screening exam designed to determine which SWR victims require an ED evaluation. Such a triage tool will assist rescuers to simultaneously honor patient autonomy and avoid risky and uninformed refusal of medical aid. Simplified medical decision-making should enable the application of this tool worldwide.

Prehosp Disaster Med 2019;34(Suppl. 1):s125-s126

doi:10.1017/S1049023X19002711

\section{Emergency Patients with Mental Crisis Accessing Emergency Medical Services in Thailand Mrs. Porntip Wachiradilok}

National Institute For Emergency Medicine 1669, Nonthaburi, Thailand

Introduction: Emergency responders face an increasing number of calls involving people with behavioral and mental crisis issue. Integrated multi-agency schemes involving ambulance, police and mental health services are now being developed to provide urgent and emergency care pathways for these vulnerable patients.

Aim: The objectives were to study the situation, characteristics, issues, and accessibility to emergency medical services (EMS) and appropriate treatment for emergency patients with a mental crisis in Thailand.
Methods: The sample included 26,511 mental crisis patients accessing EMS. Data were obtained from the database of the Information Technology for Emergency Medical System between 2015-2017 and from stakeholders from four provinces distributed regionally using focus groups and in-depth interviews. The data were analyzed using descriptive statistics and content analysis.

Results: The number of patients with mental crisis accessing EMS increased in the past three years. Most patients are male in the working age group from the Northeastern area during the raining and winter season, especially between September and October. During patient encounters with maniacal attacks, assistance will be requested from the police and the emergency medical units. The response depends on the experience and community capability. The emergency responder teams had insufficient knowledge and skills. Emergency rooms in most hospitals lack specific caring unit. Psychiatric hospitals have different criteria for admitting patients. Most had no fast track system and even refuse admittance.

Discussion: Mental crisis patient calls with EMS were rising. However, accessibility to appropriate service centers was still an issue. Most hospitals lack prioritized access and staffs had insufficient knowledge and skills. Cooperation among the police, emergency medical operation team and the rapid psychiatric emergency team is need to be reinforced.

Prehosp Disaster Med 2019;34(Suppl. 1):s126

doi:10.1017/S1049023X19002723

Emergency Response Training Program for Theme Parks: Experiences of Taiwan

Dr. Yawen Hsiau ${ }^{2}, M r$. Yu-Han Liu ${ }^{1}$, Dr. Chi-Chun Lin ${ }^{3}$

1. Disaster medicine Section,Emergency department, Taoyuan general hospital of MOHW, Taiwan, Taoyuan, Taiwan

2. Taiwan Development Association For Disaster Medical Teams, Taoyuan, Taiwan

3. Emergency Department,Ton-Yen General Hospital, Hsinchu County, Taiwan

Introduction: "Tailor-made" training programs have been started in two theme parks in North and East Taiwan after the dust explosion of Ba-xien theme park in 2015. The training programs emphasized several areas. They work to strengthen the incident command system (ICS) and the skills of first responders, especially evacuation, placement, triage, and first aid, as well as to assist the park's cooperation with local disaster response units, such as the fire department and Health Bureau.

Methods: The first step was to find out the practical problems of the two theme parks, and then make a one-year, tailor-made training program according to the needs of parks and different levels of staff: senior supervisors, middle-level district supervisors, and frontline colleagues. After the phased training, the training results are inspected in the non-scripted exercise mode.

Results: It was found that the staff are relatively familiar with the evacuation process and placement of tourists. The initial 